\title{
Identification of friction parameters for limited relative displacement contacts
}

\author{
Maxime Couillard, Patrice Masson* and Philippe Micheau \\ GAUS, Mechanical Engineering Department, Université de Sherbrooke, Sherbrooke, QC, Canada JIK $2 R 1$
}

Received 6 May 2008

Revised 2 September 2008

\begin{abstract}
Damping using dry friction has long been recognized as an effective control method for many vibration problems. However, given the strong nonlinear nature of friction, the theoretical and experimental investigations of associated non-linear control methods are much more difficult than for linear control methods. Moreover, the difficulty of identifying friction models parameters for Limited Relative Displacement (LRD) contacts is still a subject of research. This study first proposes an identification procedure to evaluate the ability of the LuGre friction model to predict small amplitude (30 $\mu \mathrm{m}-150 \mu \mathrm{m})$ frictionally damped vibrations for a LRD contact. An experimental setup implementing an ideal frictionally damped Single Degree Of Freedom (SDOF) oscillator connected to an electrodynamic shaker is then presented to study friction damping. The simulation results are assessed against the experimental results, demonstrating that the identification procedure is well suited to estimate the parameters of the LuGre friction model and that the model captures very well the friction phenomenon for small amplitude vibrations.
\end{abstract}

Keywords: Vibration control, nonlinear dynamics, damping control, dry friction, system identification

\section{Introduction}

Friction has, for a long time, been an easy and straightforward method to add passive damping to many dynamical systems [8]. For example, it has been used in gas turbines to reduce the strain that the blades undergo during flutter [19] or to design friction based isolation systems for seismic applications [18]. Moreover, it has been recognized that friction is a major source of damping in bolted joint connections of large space structures [13]. Researchers have also recently proposed varying in real-time the normal force to the friction interface in order to improve the ability of friction to reduce vibrations $[7,9,10,13,16]$, this type of control being called "semi-active". An intricate part of evaluating the effectiveness of friction damping in these applications is indeed the modeling of the friction phenomena.

Friction modeling is of interest for many researchers [3]. Extensive research has been carried out in tribology [2] but other fields such as precision motion control [6] and acoustics and vibrations [1,4] have expressed interest. Generally speaking, two broad classes of friction models can be distinguished: the constitutive and phenomenological models [19]. Constitutive models are based on contact mechanics equations and relate the stress and strain within the contact interface to determine the friction force. These types of models are very complex and are still subject of research. On the other hand, phenomenological models are based on experimental observations and provide a global relation allowing to approximate a mean friction force. The phenomenological models are far less complex and give sufficient description of the friction phenomena for many applications. They are thus usually preferred by control engineers. Within the phenomenological model class, one can further distinguish between the algebraic and

*Corresponding author. Tel.: +1 8198218000 ext. 62152; Fax: +1 819821 7163; E-mail: Patrice.Masson@ USherbrooke.ca. 


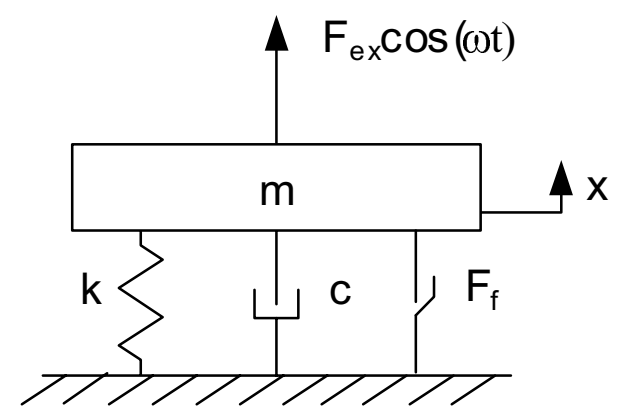

Fig. 1. Frictionally damped SDOF system.

dynamic friction classes of models [17]. Algebraic friction models relate the friction force to both the normal force and the velocity by an algebraic equation. A number of relations, used to recreate experimental observations such as stiction or Stribeck effect, can be incorporated in this type of model. But, for many applications, there is need for a friction model that better reflects the behavior of friction at near zero velocity [3]. It has been shown that the classical algebraic models cannot explain the hysteretic behavior when studying friction for nonstationary velocities, nor variations in the break-away force with the rate of application of the excitation force, nor small displacements that occur at the contact interface during stiction [6]. A friction model that has recently received a large amount of attention is the LuGre friction model developed by Canudas de Wit and co-workers [6] for precision motion control applications. The LuGre friction model is able to reproduce properties such as hysteresis, spring-like characteristics for stiction and varying break-away force. The LuGre friction model uses only one state to describe the internal friction dynamics and this relative simplicity has made the model attractive for control oriented applications.

This paper is mainly interested in Limited Relative Displacement (LRD) contacts. LRD contacts are found in situations where the relative displacement between the contact interfaces is limited by the nature of the application. For example, contact interfaces in bolted joint connections of structures are LRD contacts since the displacement between two connecting members is limited, e.g. the connecting members are intended to remain stationary. This is opposed to non-LRD contacts such as those that are commonly found in motion control applications. These contacts are created by a rotational movement (e.g. electric motor), so that they are not limited in relative displacement. This difference becomes important for identification purposes since the constant velocity measurements needed in the commonly used identification procedure are very hard to acheive for LRD contacts [5], if not impossible.

A literature review tends to indicate that there is a need for the assessment of the LuGre friction model with experimental results for LRD contacts. Indeed, many researchers have used the LuGre friction model in their theoretical analyses $[7,9,10,13,14,16]$, while often raising concerns about the difficulty of identifying some parameters [9] or the necessity of using such a complex friction model [14].

\section{Focus of the study}

The purpose of this paper is: i) to provide an identification procedure for the parameters of the LuGre friction model for LRD contacts and ii) to provide side-by-side comparisons between experimental and simulation results obtained with the LuGre friction model for LRD contacts. The vibration amplitudes are kept in a $30 \mu \mathrm{m}-150 \mu \mathrm{m}$ range so that the micro-slip behavior is significant, break-away typically being observed for relative displacements of $2 \mu \mathrm{m}-5 \mu \mathrm{m}$ for steel-to-steel junctions [3]. This study exploits the classical frictionally damped Single Degree Of Freedom (SDOF) system excited by a harmonic forcing function, as shown in Fig. 1.

The structure of this paper is as follows. Section 3 first presents the LuGre friction model and the procedure used in this paper for the identification of the parameters of this model. Section 4 presents the experimental set-up, the results obtained for the identification of the parameters of the LuGre friction model, and the experimental assessment of the friction model. Finally, Section 5 concludes this study and discusses future work. 


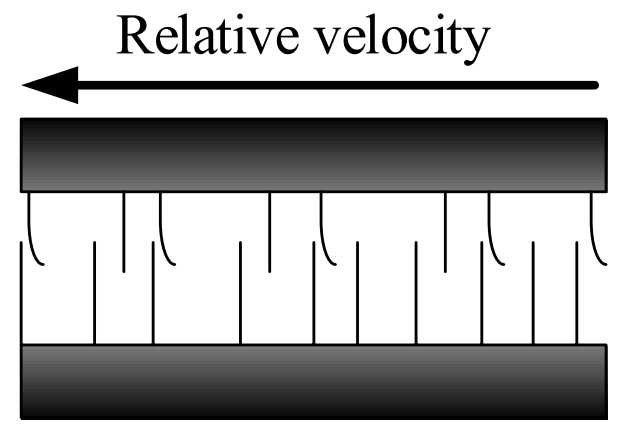

Fig. 2. Bristles-based representation of the friction interface.

\section{Identification of the LuGre friction model parameters}

\subsection{The LuGre friction model}

The LuGre friction model is based on a "bristles" representation of the friction at the interface of two rigid bodies having a relative velocity $\dot{x}$, as illustrated in Fig. 2 [6]. Taking $z$ as the average bristles deflection and thus the internal friction state, the model can be described using two equations. The first one describes the bristles dynamics:

$$
\frac{d z}{d t}=\dot{x}-\sigma_{0} \frac{|\dot{x}|}{g(\dot{x})} z
$$

where $\sigma_{0}$ represent the bristles stiffness and $g(\dot{x})$ is a function of the velocity, defined as:

$$
g(\dot{x})=F_{c}+\left(F_{s}-F_{c}\right) \exp \left(-\frac{\dot{x}^{2}}{v_{s}^{2}}\right),
$$

where $F_{c}$ is the Coulomb friction level, $F_{s}$ is the static friction level and $v_{s}$ is the Stribeck velocity.

The second equation describes the friction force generated by the bending of the bristles and considering a viscous friction coefficient:

$$
F_{f}=\gamma_{N}\left(\sigma_{0} z+\sigma_{1} \frac{d z}{d t}+\sigma_{2} \dot{x}\right)
$$

where $\gamma_{N}$ is the normal force coefficient, $\sigma_{1}$ represents the damping introduced by the bending of the bristles and $\sigma_{2}$ is a viscous damping coefficient introduced to account for viscous friction.

It should be noted here that, as compared to the usual representation of the LuGre friction model [9], the normal force does not appear explicitly in the preceding equations. Instead, the parameter $\gamma_{N}$ is used here, which is defined as the normal force coefficient. The rationale behind this choice is to avoid the assumption of linearity between the normal force and the friction force. The normal force is not required and thus, not measured in the proposed model; instead the parameter $\gamma_{N}$ is identified as another parameter in Eq. (3). This is further explained in Section 3.3.

The proposed identification procedure for the parameters of the LuGre friction model is as follows:

1. Identification of the dynamic parameters of the model : $\gamma_{N} \sigma_{0}$ and $\gamma_{N} \sigma_{1}$. This identification is conducted in the frequency domain.

2. Identification of the static parameters of the model: $\gamma_{N}, F_{s}, \sigma_{2}$ and $v_{s}$ while setting $F_{c}=1 \mathrm{~N}$. The identification of these parameters is conducted using an optimization-based algorithm. The reason why $F_{c}$ is held fixed during this process is further explained in Section 3.3. 


\subsection{Identification of the dynamic parameters}

The difficulty of identifying the LuGre friction model dynamic parameters arises from the fact that the internal state of the friction interface, noted as $z$, cannot be directly measured. The common method found in the literature is then to use a frequency domain identification with the friction interface in pre-sliding regime [11], which implies neglecting the second part of Eq. (1) so that $z=x$. By using this approximation, one can then consider that the friction interface behaves like a slightly damped mechanical stiffness.

For the frictionally damped SDOF oscillator, the original equation of motion of the system is:

$$
F_{e x}=m \ddot{x}+c \dot{x}+k x+F_{f},
$$

where $F_{e x}$ represents the external force, $F_{f}$ represents the friction force and $m, k$ and $c$ represent respectively the mass, stiffness and viscous damping of the system. Then, using the LuGre friction model equations, Eqs (1)-(3), considering $z=x$, the equation of motion of the system in pre-sliding regime can be written:

$$
F_{e x}=m \ddot{x}+\left(c+\gamma_{N}\left(\sigma_{1}+\sigma_{2}\right)\right) \dot{x}+\left(k+\gamma_{N} \sigma_{0}\right) x .
$$

For a harmonic displacement, the Frequency Response Function (FRF) of the system can then be written as:

$$
\frac{X(j \omega)}{F_{e x}(j \omega)}=\frac{1}{-m \omega^{2}+\left(c+\gamma_{N} \sigma_{1}+\gamma_{N} \sigma_{2}\right) j \omega+\left(k+\gamma_{N} \sigma_{0}\right)} \text {. }
$$

By assuming that $\sigma_{2} \ll \sigma_{1}$, which, for a dry friction contact is considered to be a realistic assumption [11], Eq. (6) can be expressed as:

$$
\frac{X(j \omega)}{F_{e x}(j \omega)}=\frac{1}{-m \omega^{2}+\left(c+\gamma_{N} \sigma_{1}\right) j \omega+\left(k+\gamma_{N} \sigma_{0}\right)} .
$$

Thus, a second-order model identification procedure can be used using an experimental FRF of the system to obtain $\left(k+\gamma_{N} \sigma_{0}\right)$ and $\left(c+\gamma_{N} \sigma_{1}\right)$. Once $k$ and $\gamma_{N}$ are known, $\sigma_{0}$ and $\sigma_{1}$ can thus be estimated.

\subsection{Identification of the static parameters}

As mentioned in Section 2, constant velocity measurements, which are commonly used for the identification of the LuGre friction model static parameters for non-LRD contacts, are often very difficult to achieve, if not impossible, for LRD contacts. An interesting alternative based on an optimization algorithm has been proposed by Gaul and co-workers [9] for the identification of the LuGre friction model static parameters. The approach used herein is a slightly modified version of their approach, the main difference being that the identified parameter $\gamma_{N}$ is used to isolate the influence of the normal force instead of using directly the measured normal force. In this work, the normal force is not measured and the parameter $\gamma_{N}$ is obtained through an identification procedure, as for the other parameters in Eq. (3). The advantage of this method is that the linearity assumption between the normal force and its influence on the friction force is no longer a constraint.

The identification of the static parameters is based on a multi-variables non-linear optimization algorithm that minimizes the sum of the errors between the simulated and the experimental friction force. Unicity of the solution is assumed. In order to simplify the identification procedure, the tests are conducted with the system under harmonic excitation. The optimization algorithm is based on the minimization of a cost function $J(\theta)$ representative of an error model such as:

$$
J(\theta)=\sum_{i=1}^{N}\left(F_{f}\left(t_{i}\right)-\hat{F}_{f}\left(t_{i}, \theta\right)\right)
$$

where $N$ represents the number of points per cycle, $F_{f}\left(t_{i}\right)$ is the measured friction force, and $\hat{F}_{f}\left(t_{i}, \theta\right)$ represents the simulated friction force. The vector $\theta$ represents the LuGre friction model static parameters to be identified such as:

$$
\theta=\left[\begin{array}{llll}
\gamma_{N} & F_{S} & \sigma_{2} & v_{s}
\end{array}\right]
$$

The identification procedure of the LuGre friction model static parameters is presented as a flow chart in Fig. 3. Note that because of the non-linear nature of the cost function, a gradient-based optimization algorithm might lead to a local minima. The performance of the approach thus relies on proper initial estimates provided to the algorithm. In this study, this has been done by visual inspection of the steady-state regime of the system under harmonic excitation. 
Table 1

LuGre friction model parameters used for simulation

\begin{tabular}{ccccccc}
\hline$\gamma_{N}$ & $F_{c}$ & $F_{s}$ & $\sigma_{0}$ & $\sigma_{1}$ & $\sigma_{2}$ & $v_{s}$ \\
$(\mathrm{~N})$ & $(\mathrm{N})$ & $(\mathrm{N})$ & $(1 / \mathrm{m})$ & $(\mathrm{s} / \mathrm{m})$ & $(\mathrm{s} / \mathrm{m})$ & $(\mathrm{m} / \mathrm{s})$ \\
\hline 1.5 & 1 & 1.2 & $1 \times 10^{7}$ & 200 & 1 & 0.05 \\
\hline
\end{tabular}

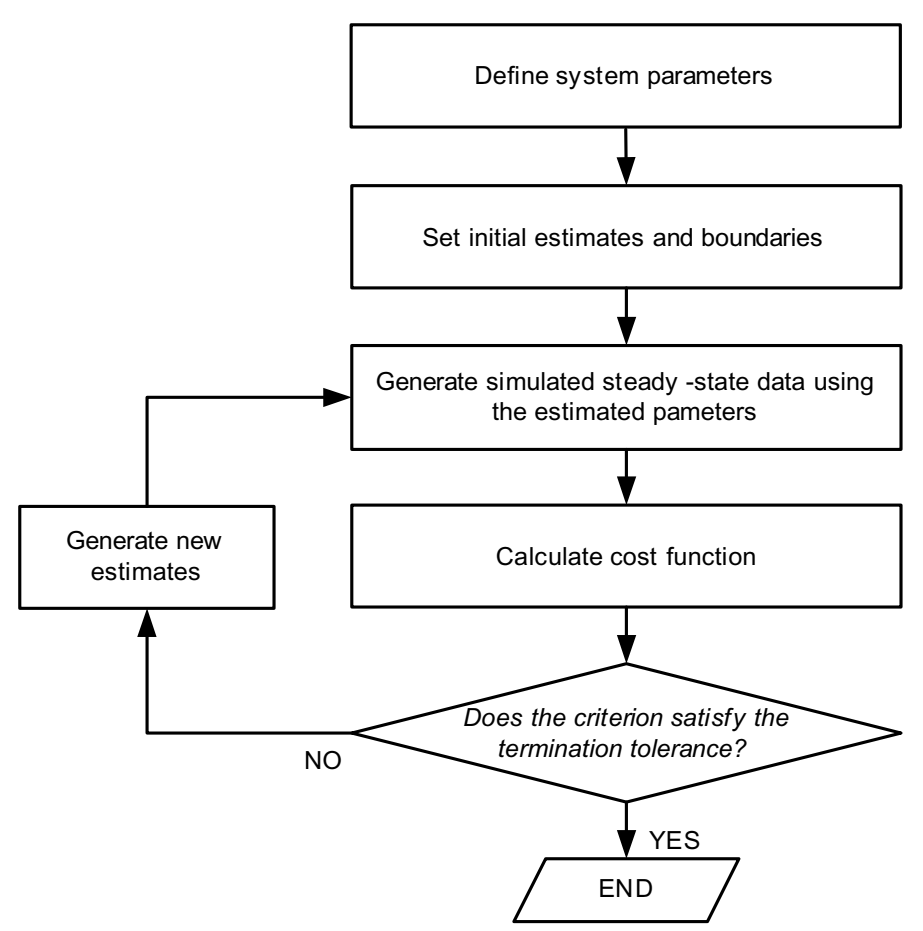

Fig. 3. Optimization-based algorithm for the identification of the LuGre friction model static parameters.

\subsection{Simulation results}

A simulation is conducted in order to validate the identification procedure presented in Sections 3.2 and 3.3. The LuGre friction model is implemented in MATLAB/Simulink and is first used to generate steady-state data, using the parameters of Table 1 considered as the true values. These simulated results are supplied to the optimization algorithm (Fig. 3) implemented in MATLAB in order to provide simulated experimental data: "measured friction force" and "measured movements". Then, the optimization algorithm is started with false values of estimated parameters. At each iteration, the algorithm calls the LuGre friction model to obtain the steady-state response for the given estimated parameters. Figure 4 shows a typical result were the identified parameters have converged to the true ones after 150 iterations. The simulations suggest that the approach can lead to very good results for initial estimates within $50 \%-150 \%$ of the true parameter value. Hence, this illustrates that the minimization of the cost function $J(\theta)$ ensures proper convergence of the estimated parameters $\theta$ to the exact parameters.

\section{Experimental study}

\subsection{Experimental set-up}

An exploded view of the semi-active friction device developed by Buaka [4] and used for experimental investigation is shown in Fig. 5. Two friction pads (1) are pushed on a moving mass (2) which is supported by two thin steel sheets (3) providing the spring force. The moving mass is made out of AISI 1018 steel and the friction pads are 

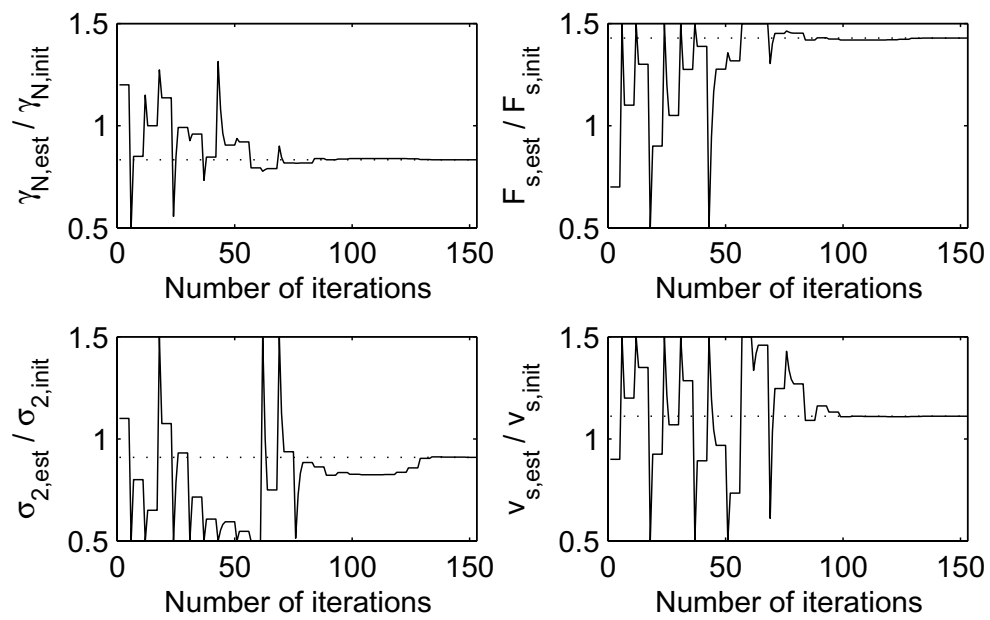

Fig. 4. Simulation of the identification of the LuGre friction model static parameters using the optimization-based algorithm ( - ) and true parameter value $(\cdots)$.

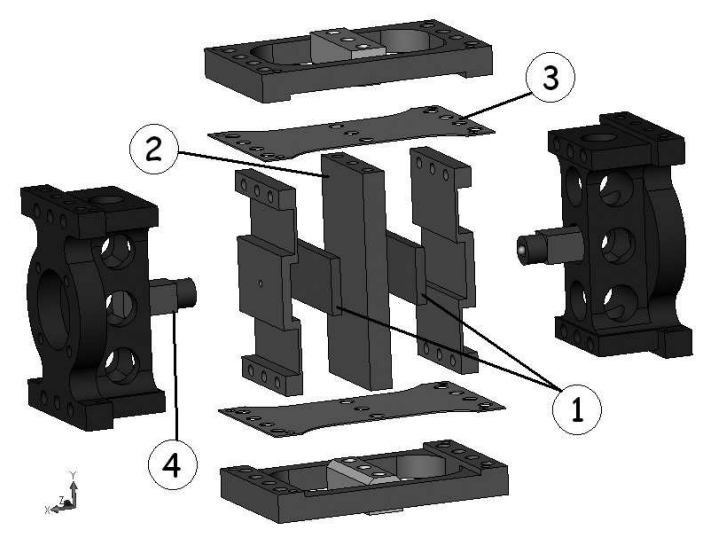

Fig. 5. CAD model of the friction damper.

cut from automotive carbon brake pads. Even if such materials are usually used for sliding contact applications, they are still appropriate for bristles-based applications due to the contact plateaux at the contact surfaces. The electrodynamic shaker is directly attached to the moving mass through a stinger. Two piezoelectric stack actuators (4) are also incorporated in the design to allow real-time variation of the normal force. Note that the piezoelectric stack real-time actuation capabilities were not used for the purpose of this paper.

Two different vibration shakers are used for the experiment, one Br üel and Kjaer 4809 shaker and one MB Dynamics Modal 50A shaker. The force and acceleration are measured with a PCB Piezotronics $288 D 01$ impedance head, FRFs are obtained using a Hewlett-Packard 35665a dual channel analyzer and time data acquisition is performed using a $d S P A C E$ system. A picture of the experimental set-up is shown in Fig. 6 and a block diagram is presented in Fig. 7. Note that since the sampling rate $(10 \mathrm{kHz})$ is very fast in comparison with the fundamental frequency of the excitation $(20-40 \mathrm{~Hz})$, no anti-aliasing filters are used with the $A S P A C E$ system.

The friction force is obtained indirectly from the measurement of the velocity $\dot{x}$, the position $x$, and the excitation force $F_{e x}$ using the following expression:

$$
F_{f}=-m \ddot{x}-c \dot{x}-k x+F_{e x}
$$

The velocity and the position are obtained by integration of the acceleration signal. These integrations are made off-line in order to avoid the delays introduced by the necessary highpass filters for real-time integration. 


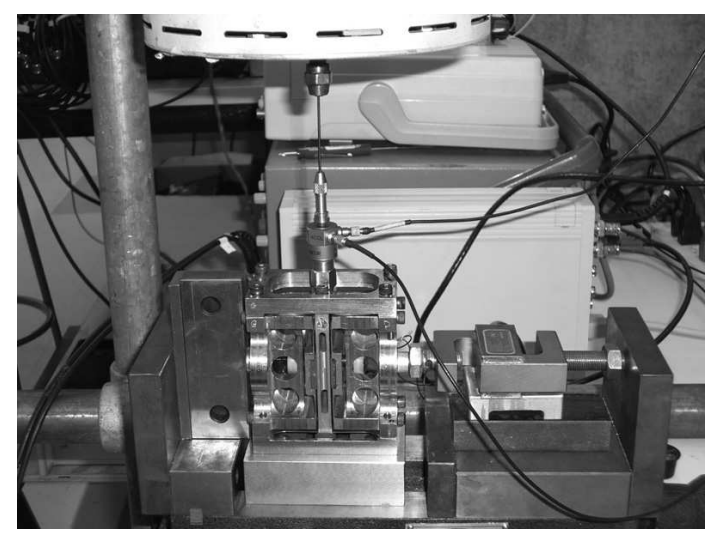

Fig. 6. Picture of the experimental set-up.

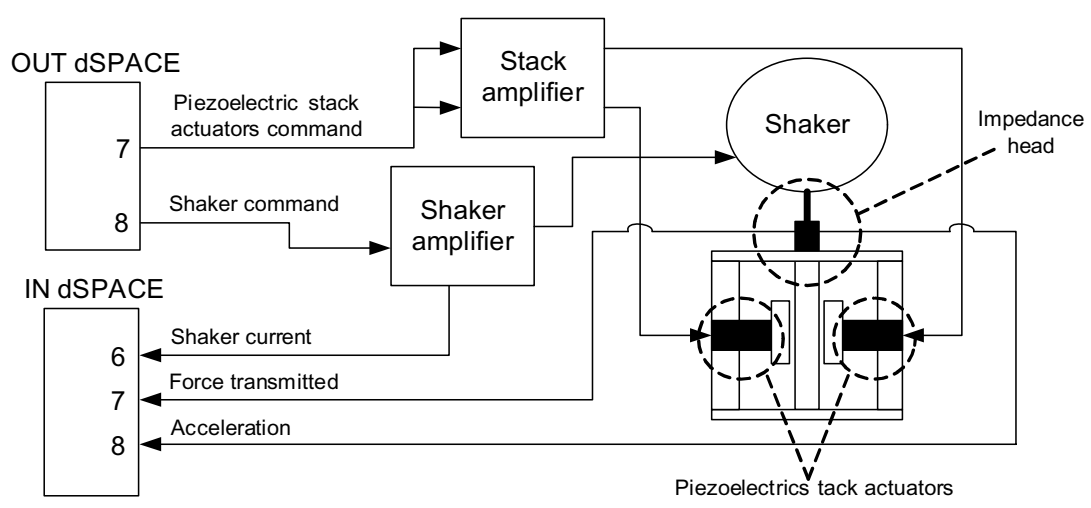

Fig. 7. Block diagram of the experimental set-up.

\subsection{Harmonic excitation of the friction damper}

Harmonic excitation of a linear system using an electrodynamic shaker is somewhat trivial due to the fact that the response of the linear system is also of harmonic type. One can thus use the electrodynamic shaker in either voltage or current control mode and use either a fixed or suspended base configuration to obtain a harmonic force measured at the force transducer. But the harmonic forcing of a frictionally damped SDOF system, or any nonlinearly damped system, is not as trivial.

Consider a SDOF system connected to a fixed based electrodynamic shaker as illustrated in Fig. 8, where subscript $a$ denotes the dynamic properties of the armature and subscript $d$ denotes the dynamic properties of the device. The total system, composed of the device and the armature, is essentially another SDOF system with dynamic properties such as: $m=m_{a}+m_{d}, c=c_{a}+c_{d}$ and $k=k_{a}+k_{d}$. The force transmitted to the device and measured at the impedance head can then be expressed as:

$$
F_{t}=-m_{d} \ddot{x}-c_{d} \dot{x}-k_{d} x-F_{f} .
$$

A harmonic excitation of the device is sought for the purpose of this study. However, comparing Eqs (10) and (11) indicates clearly that there is no guarantee that the force measured at the impedance head, $F_{t}$, will be of the same form as the command signal to the shaker, $F_{e x}$. In fact, unless the assumption is made that the dynamic properties of the armature are negligible with regards to those of the device, it is not possible to apply a harmonic forcing function to a frictionally damped SDOF through the conventional use of an electrodynamic shaker.

One way to go around this problem is to use the current injected to the electrodynamic shaker instead of the force measured by the impedance head to determine the total force applied to the combined system (armature and device). 


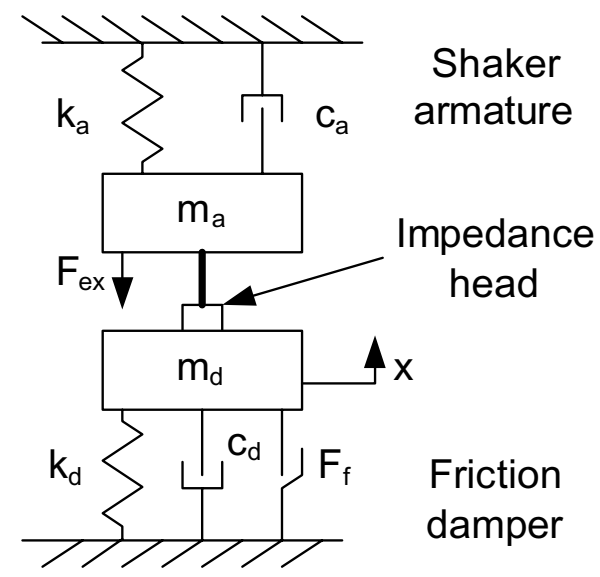

Fig. 8. Model of the shaker armature and the friction damper.

This is based on the fact that the current in the shaker is related to the force generated by the well known Amp ère's law [15]:

$$
F_{e x}=(B l n) I
$$

where $B$ is the field strength, $l$ is the length of one coil, $n$ is the number of coils and $I$ is the conductor current. In current control mode, the force injected in the combined system will then be of the same form as the command input waveform. Indeed, the dynamic properties of the experimental set-up will now include the armature dynamics properties but if one is interested solely in theoretical analysis of the harmonically excited friction damped SDOF system, this should not be a problem. Thus, this method has been used to obtain the experimental results presented in this section.

\subsection{Identification of the SDOF system}

This section presents the procedure for the identification of the dynamic properties (mass, damping and stiffness) of the experimental set-up. This is required to ultimately measure the friction force and to simulate the frictionally damped SDOF system.

The dual channel analyzer was used to obtain the accelerance transfer function between the input current to the shaker to the accelerometer signal of the impedance head installed on the moving mass without any friction force applied. Two different vibration shakers were used for the experiments in order to validate that the friction model parameters to be identified in Section 4.4 are independent of the vibration shaker, and thus independent of the system parameters determined without friction. The parameters of the SDOF system obtained using a second-order identification procedure are summarized in Table 2. The impedance head is used to measure the input force for the identification of the parameters for the friction damper only. A typical side-by-side comparison of the experimental accelerance transfer function and the theoretical accelerance transfer function obtained using the identified parameters is shown in Fig. 9 for the set-up with the MB Dynamics Modal 50A shaker. The agreement between the experimental and the theoretical FRFs is excellent.

\subsection{Identification of the LuGre friction model parameters}

The steps for the complete identification of the LuGre friction model parameters presented in Sections 3.2 and 3.3 are recalled here:

1. Obtain a FRF in pre-sliding regime. To do this, one must make sure that the maximum relative displacement is small compared to the break-away relative displacement;

2. Run a second-order model identification procedure with the parameter $m$ previously identified. This allows to obtain $\left(k+\gamma_{N} \sigma_{0}\right)$ and $\left(c+\gamma_{N} \sigma_{1}\right)$ 
Table 2

Identified parameters for the SDOF systems, without any friction force

\begin{tabular}{|c|c|c|c|c|c|}
\hline Set-up & $\begin{array}{c}m \\
(\mathrm{~kg})\end{array}$ & $\begin{array}{c}k \\
(\mathrm{~N} / \mathrm{m})\end{array}$ & $\begin{array}{c}c \\
(\mathrm{Ns} / \mathrm{m})\end{array}$ & $\begin{array}{c}\omega_{n} \\
(\mathrm{~Hz})\end{array}$ & $\begin{array}{c}\zeta \\
(\%)\end{array}$ \\
\hline Friction damper only & 0.1926 & 8258 & 0.6919 & 32.96 & 0.87 \\
\hline Friction damper with Brüel and Kjaer 4809 shaker & 0.2709 & 17321 & 40.1 & 40.24 & 29.27 \\
\hline Friction damper with $M B$ Dynamics Modal $50 A$ shaker & 0.4578 & 10327 & 2.41 & 23.9 & 1.75 \\
\hline
\end{tabular}

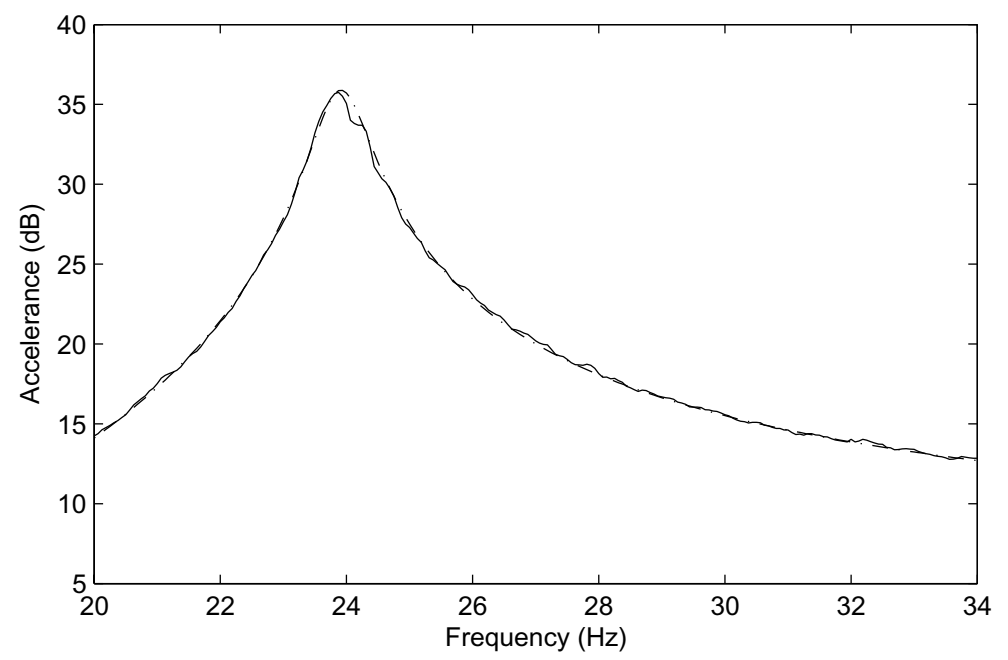

Fig. 9. Experimental and theoretical frequency response functions for the set-up with the MB Dynamics Modal 50A shaker, without any friction force. Experimental frequency response function $(-)$ and theoretical frequency response function from identification $(\cdot-\cdot)$.

3. Subtract the parameters $k$ and $c$ previously identified in order to obtain $\gamma_{N} \sigma_{0}$ and $\gamma_{N} \sigma_{1}$;

4. Run the optimization-based static parameters identification algorithm in order to determine $\theta=$ $\left[\begin{array}{llll}\gamma_{N} & F_{S} & \sigma_{2} & v_{s}\end{array}\right]$

The pre-sliding regime FRF obtained to identify the LuGre friction model dynamic parameters is shown in Fig. 10. The FRF was obtained applying a white noise with a RMS amplitude of $0.035 \mathrm{~N}$, while the static friction force was evaluated at around $1.5 \mathrm{~N}$. It can be seen that there is a well-defined resonance in the vicinity of $1200 \mathrm{~Hz}$. Assuming that this resonance is caused by the bristles dynamics, the second-order model identification procedure yields the parameters presented in Table 3. Other resonances seen in Fig. 10 might be associated to the dynamic behavior of the thin sheets used to support the moving mass or the support of the stack actuators.

For the identification of the LuGre friction model static parameters, a harmonic forcing function was generated by the shaker in order to obtain a stick-slip type of response by the friction damper. A stick-slip regime allows to get a clear visual representation of the bristles because it introduces a phenomena called micro-slip oscillations [12], which can be related to the bristles stiffness and damping. Figure 11 presents the result for the optimization based algorithm for the identification of the LuGre friction model static friction parameters. The complete identified LuGre friction model parameters are presented in Table 4 and the comparison between the experimental and simulated time domain acceleration and friction force signals is presented in Fig. 12. Clearly, the LuGre friction model can reproduce very accurately the friction phenomenon. The micro-slip oscillations due to the bristles dynamics are well reproduced.

In order to validate that the LuGre friction model parameters hold for other conditions, two other tests were conducted. First, Fig. 13 presents the results obtained when keeping the same test set-up and forcing amplitude parameters as before but using a different normal force at the friction interface. It can be seen that by simply adjusting $\gamma_{N}$, the friction phenomenon is well predicted. Note that the micro-slip oscillations have disappeared since there is no apparent stiction in the motion.

The second test was done using a different electrodynamic shaker in order to ensure that the results were not dependent on the choice of the shaker. The test was also conducted under different frequency and forcing amplitude 
Table 3

Results from the identification procedure for the LuGre friction model dynamic parameters

\begin{tabular}{cc}
\hline$\gamma_{N} \sigma_{0}$ & $\gamma_{N} \sigma_{1}$ \\
$(\mathrm{~N} / \mathrm{m})$ & $(\mathrm{Ns} / \mathrm{m})$ \\
\hline $1.0106 \times 10^{7}$ & 134.41 \\
\hline
\end{tabular}

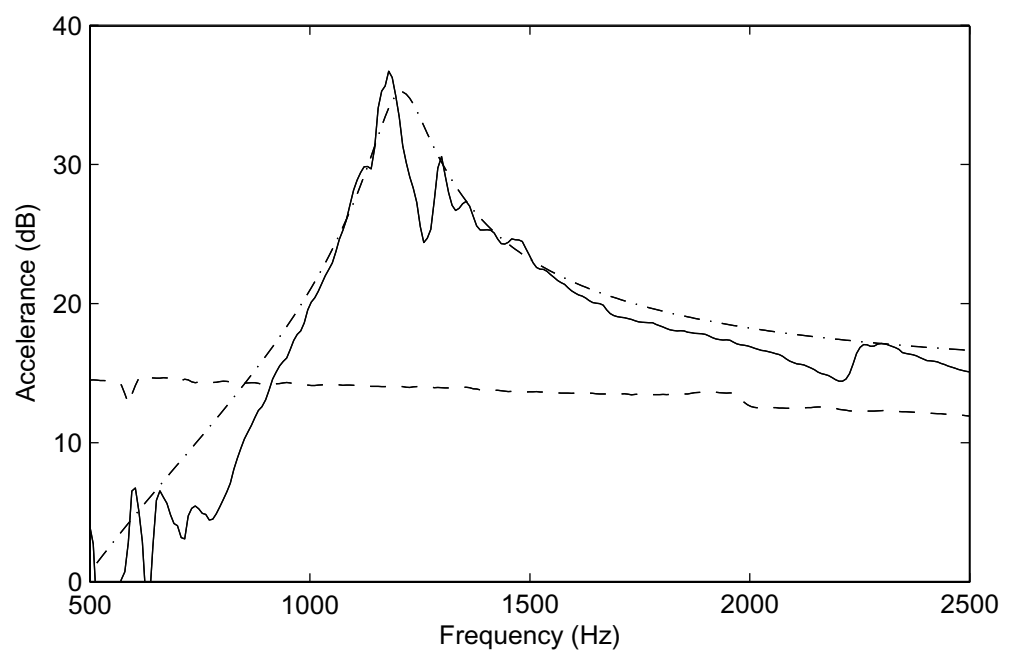

Fig. 10. Frequency domain identification of the LuGre friction model dynamic parameters for the set-up with the Brüel and Kjaer 4809 shaker. Experimental frequency response function $(-)$, theoretical frequency response function from identification $(\cdot-\cdot)$ and experimental frequency response function without friction force applied (- - -).
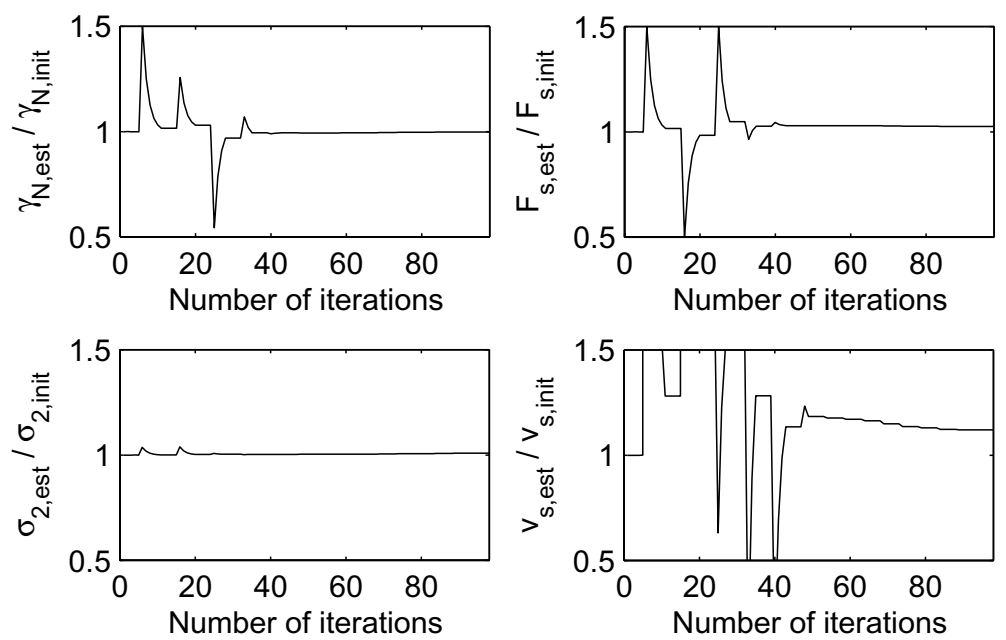

Fig. 11. Results from the identification of the LuGre friction model static parameters using the optimization-based algorithm.

parameters. Again, the friction force was adjusted to obtain a clear representation of the bristles dynamics with the micro-slip oscillations. Figure 14 presents the acceleration and friction force signals obtained. By simply adjusting the parameter $\gamma_{N}$, the friction phenomenon is once again accurately predicted. Changing the shaker had, in this case, the effect of modifying the normal force, and thus $\gamma_{N}$. 
Table 4

Identified parameters for the LuGre friction model

\begin{tabular}{cccccc}
\hline$F_{c}$ & $F_{s}$ & $\sigma_{0}$ & $\sigma_{1}$ & $\sigma_{2}$ & $v_{s}$ \\
$(\mathrm{~N})$ & $(\mathrm{N})$ & $(1 / \mathrm{m})$ & $(\mathrm{s} / \mathrm{m})$ & $(\mathrm{s} / \mathrm{m})$ & $(\mathrm{m} / \mathrm{s})$ \\
\hline 1 & 1.2312 & $8.5247 \times 10^{6}$ & 101.28 & 0.001 & 0.0224 \\
\hline
\end{tabular}


Fig. 12. Time history of the acceleration and the friction force for the set-up with the Brüel and Kjaer 4809 shaker with $F_{\text {ex }}=1.75 \mathrm{~N}$, $\omega=35 \mathrm{~Hz}$ and $\gamma_{N}=1.297 \mathrm{~N}$. Experimental time history $(-)$ and simulated time history $(\cdot-\cdot)$.
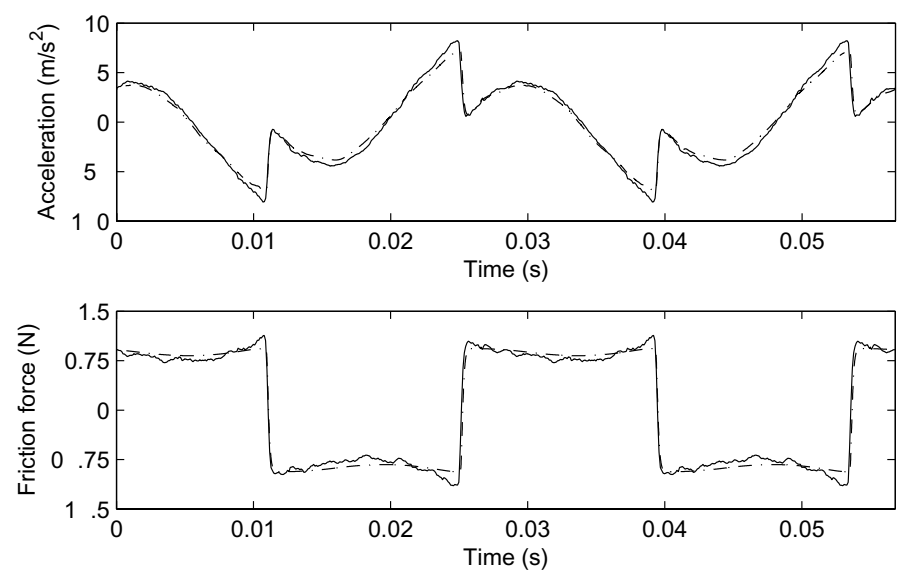

Fig. 13. Time history of the acceleration and the friction force for the set-up with the Brïel and Kjaer 4809 shaker with $F_{\text {ex }}=1.75 \mathrm{~N}$, $\omega=35 \mathrm{~Hz}$ and $\gamma_{N}=0.760 \mathrm{~N}$. Experimental time history $(-)$ and simulated time history $(\cdot-\cdot)$.

\section{Conclusion}

\subsection{Summary}

This paper focused on dry friction contacts that are found in situations where the relative displacement between the contact interfaces is limited, these contact are termed LRD contacts. The ability of the LuGre friction model to predict the friction phenomenon for small displacements LRD contacts is evaluated for the theoretical case of the frictionally damped SDOF system.

A complete identification procedure for both the dynamic and static LuGre friction model parameters is presented. Since the classical constant velocity measurements method is difficult to apply for LRD contacts, an optimizationbased algorithm is used, which is essentially a modified version of the algorithm presented by Gaul and co-workers [9]. 

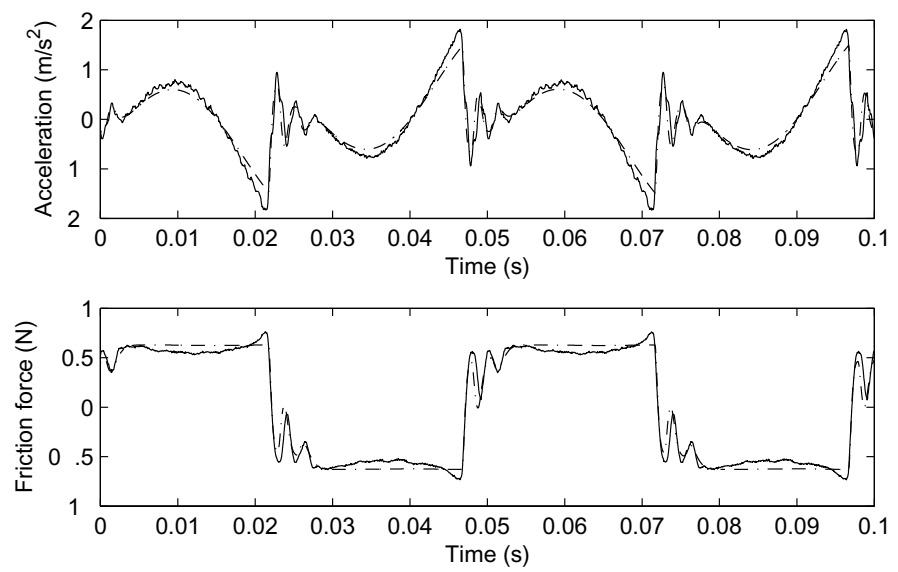

Fig. 14. Time history of the acceleration and the friction force for the set-up with the MB Dynamics Modal 50A shaker with $F_{\text {ex }}=0.75 \mathrm{~N}$, $\omega=20 \mathrm{~Hz}$ and $\gamma_{N}=0.511 \mathrm{~N}$. Experimental time history $(--)$ and simulated time history $(\cdot-\cdot)$.

Side-by-side comparisons between experimental and simulation results, obtained with the LuGre friction model, are presented. It is concluded that for small displacement LRD contacts friction modelling, the use of the LuGre friction model allows an adequate representation of the dynamics of the phenomenon.

\subsection{Future work}

In order to better assess the robustness of the identification procedure presented in this paper, a sensitivity and convergence analysis would need to be conducted. It would also be necessary to further investigate the relation between the normal force, $F_{N}$, and the normal force coefficient, $\gamma_{N}$, defined in this paper. The analysis will also be extended using friction pads materials other than automotive pads.

Work is currently being carried out to develop bristles-based semi-active control schemes and validate the concepts by making use of the piezoelectric stack actuators incorporated in the semi-active friction device. This is conducted with the ultimate goal to provide tools for the optimization of structural friction damping.

\section{Acknowledgments}

This work has been supported by the "Fonds Québécois de la Recherche sur la Nature et les Technologies" (FQRNT) and by the "Natural Sciences and Engineering Research Council" (NSERC).

\section{References}

[1] A. Akay, Acoustics of friction, Journal of the Acoustical Society of America 111(4) (2002), 1525-1548.

[2] S. Andersson, A. Soderberg and S. Bjorklund, Friction models for sliding dry, boundary and mixed lubricated contacts, Tribology International 40(4) (2007), 580-587.

[3] B. Armstrong-Helouvry, P. Dupont and C. Canudas De Wit, Survey of models, analysis tools and compensation methods for the control of machines with friction, Automatica 30(7) (1994), 1083-1138.

[4] P. Buaka, P. Masson and P. Micheau, Determination of normal force for optimal energy dissipation of harmonic disturbance in a semi-active device, Journal of Sound and Vibration 311(3) (2008), 633-651.

[5] P. Buaka Muanke, Development of a semi-active device for the reduction of vibrations in mechanical structures by dry friction energy dissipation, $\mathrm{PhD}$ thesis, Université de Sherbrooke, 2005, (in French).

[6] C. Canudas de Wit, H. Olsson, K.J. Astrom and P. Lischinsky, New model for control of systems with friction, IEEE Transactions on Automatic Control 40(3) (1995), 419-425.

[7] P. Dupont, P. Kasturi and A. Stokes, Semi-active control of friction dampers, Journal of Sound and Vibration 202(2) (1997), 203-218.

[8] A.A. Ferri, Friction damping and isolation systems, Journal of Mechanical Design, Transactions of the ASME 117B (1995), 196-206. 
[9] L. Gaul, H. Albrecht and J. Wirnitzer, Semi-active friction damping of large space truss structures, Shock and Vibration 11(3-4) (2004), $173-186$.

[10] E. Guglielmino and K.A. Edge, A controlled friction damper for vehicle applications, Control Engineering Practice 12(4) (2004), 431-443.

[11] R.H.A. Hensen, M. Rene, J.G. Van De Molengraft and Maarten Steinbuch. Frequency domain identification of dynamic friction model parameters, IEEE Transactions on Control Systems Technology 10(2) (2002), 191-196.

[12] N. Hinrichs, M. Obstreich and K. Popp, On the modelling of friction oscillators, Journal of Sound and Vibration 216(3) (1998), 435-459.

[13] J.S. Lane, A.A. Ferri and B.S. Heck, Vibration control using semi-active friction damping, volume 49, Anaheim, CA, USA, 1992, 165-171. ASME, Design Engineering Division.

[14] M. Lorenz, B. Heimann and V. Hartel, A novel engine mount with semi-active dry friction damping, Shock and Vibration 13(4-5) (2006), $559-571$.

[15] K.G. McConnell, Vibration Testing: Theory and Practice, first edition, Wiley, 1995.

[16] R. Nitsche and L. Gaul, Smart friction driven systems, Smart Materials and Structures 14(1) (2005), 231-236.

[17] H. Olsson, K.J. Astrom, C. Canudas De Wit, M. Gafvert and P. Lischinsky, Friction models and friction compensation, European Journal of Control 4(3) (1998), 176-195.

[18] A. Pirrotta and R.A. Ibrahim, Experimental investigation of friction-base isolation, Probabilistic Engineering Mechanics 12(3) (1997), $125-136$.

[19] K. Popp, L. Panning and W. Sextro, Vibration damping by friction forces: Theory and applications, Journal of Vibration and Control 9(3-4) (2003), 419-448. 

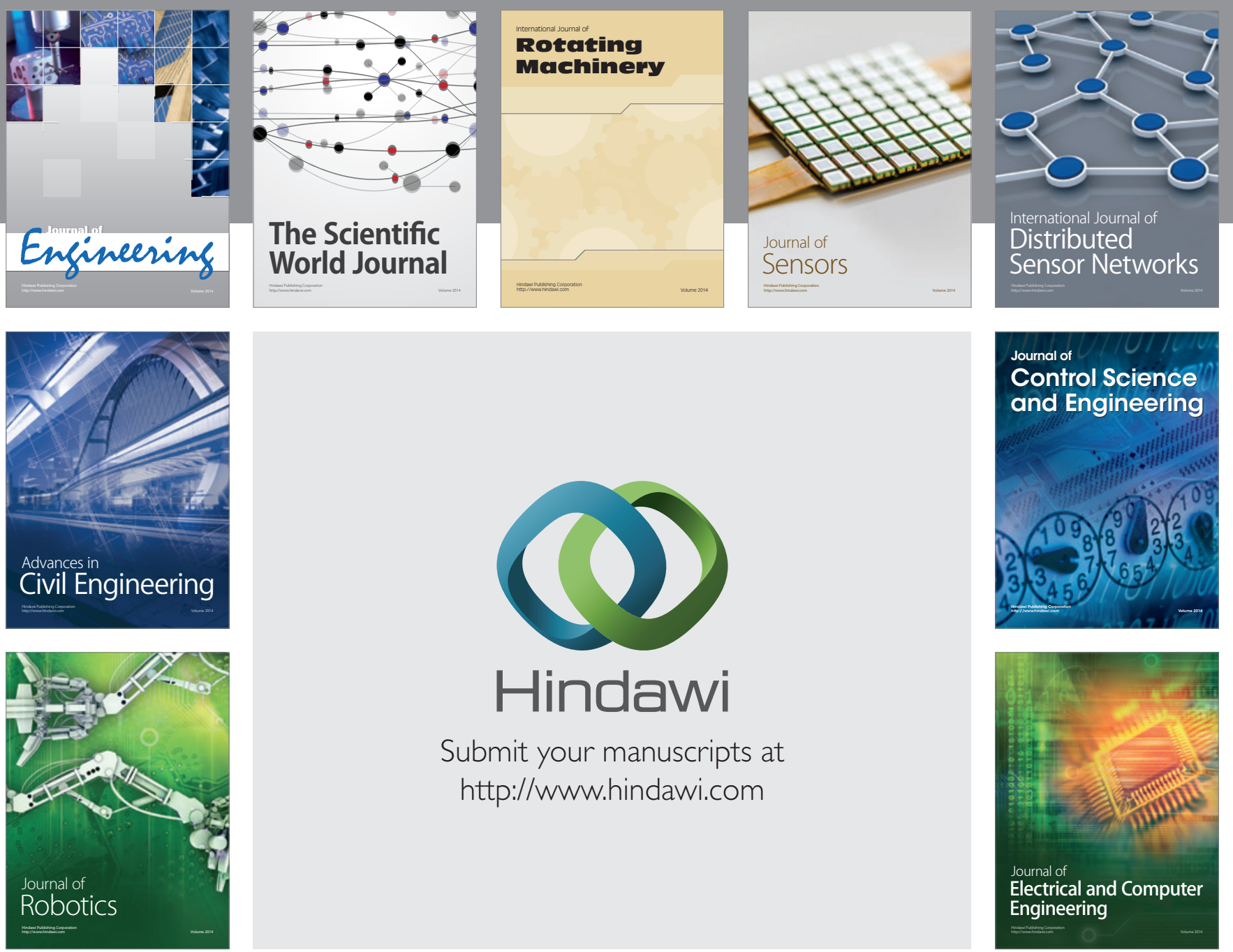

Submit your manuscripts at

http://www.hindawi.com
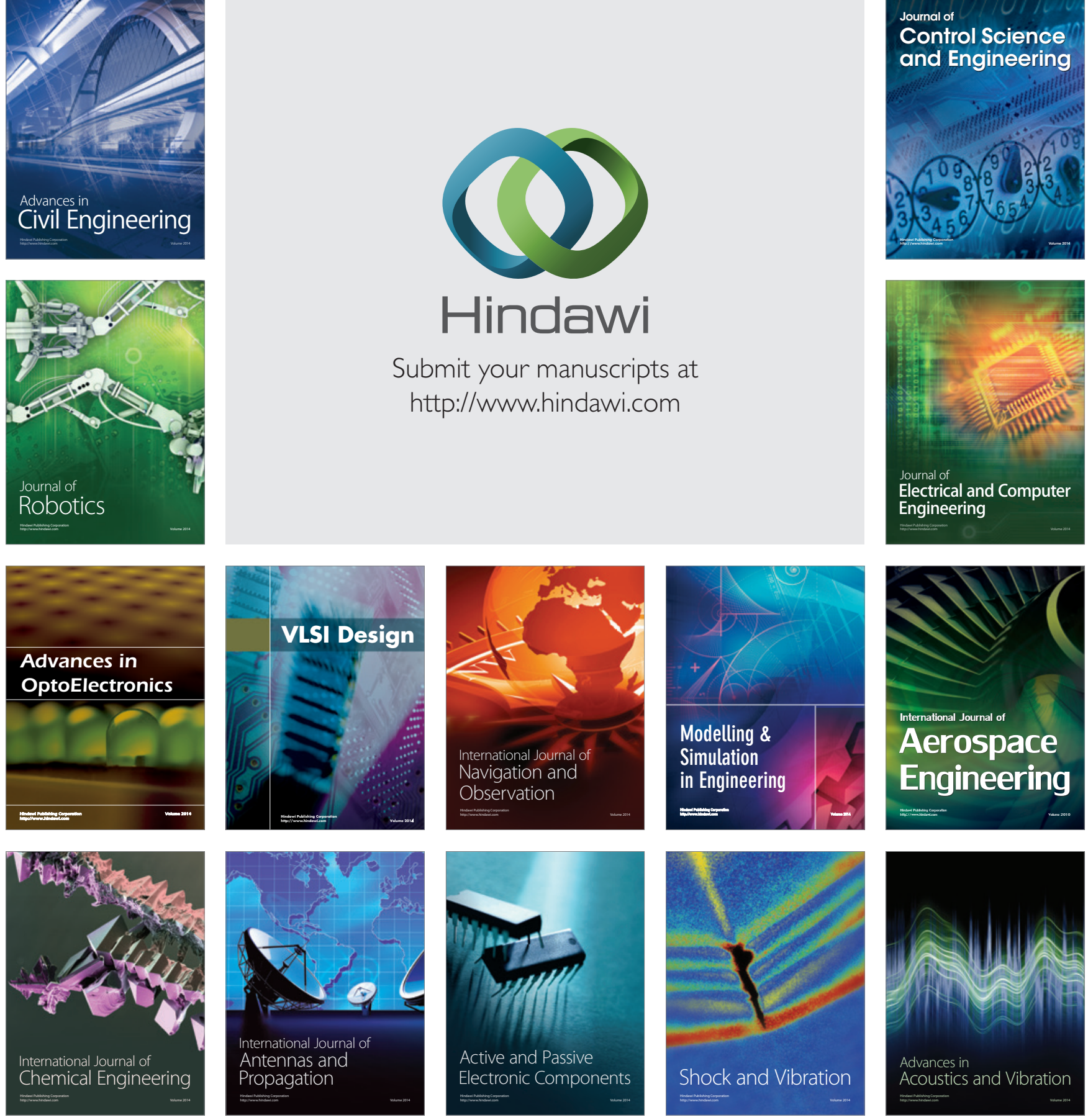